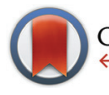

CrossMark

\&lick for updates

Cite this: Polym. Chem., 2014, 5, 6651

Received 30th June 2014,

Accepted 12th August 2014

DOI: 10.1039/c4py00911h

www.rsc.org/polymers

\section{Reactive imidazole intermediates: simplified synthetic approach to functional aliphatic cyclic carbonates $\uparrow$}

\author{
Johan V. Olsson, Daniel Hult, Yanling Cai, Sandra García-Gallego and \\ Michael Malkoch*
}

\begin{abstract}
Reactive imidazole intermediates based on $A B_{2}$ and $A_{3}$ monomers, i.e. bis(methylol) propionic acid (bis-MPA) and trimethylolpropane (TMP) have successfully been synthesized and isolated on a 100 gram scale via a facile synthetic protocol using 1,1'-carbonyldiimidazole (CDI) as a key reagent. The robustness of the imidazole intermediates as bench stable precursors enabled the synthesis of a library of functional cyclic carbonates bearing relevant functionalities including hydrophilic PEGs, bioactive cholesterol and clickable groups. A number of functional polycarbonates were obtained by ring-opening polymerization, and their relevance in biomedical applications was highlighted by their low cytotoxicity on human dermal fibroblasts (hDF).
\end{abstract}

With the apparent demand for functional polymers needed to fulfil the criteria set by a modern society, scientists, only limited by their imagination, are constantly proposing novel architectures. Polycarbonates (PCs), particularly functional aliphatic polycarbonates (FAPCs) with pendant reactive groups along the main chain, have recently received significant attention due to their envisioned use in biomedical applications. ${ }^{1}$ An attractive feature of PCs, in comparison with polyesters, is their biodegradation profile that proceeds without concomitant acid formation. ${ }^{2}$ In contrast, biodegradation of polyesters may lead to high local concentrations of acid in vivo ${ }^{3}$ which could impact acid-labile drug-delivery systems. The utility of FAPCs ranges from being promising candidates as artificial biomaterials to acting as passive drug delivery systems. ${ }^{4}$ Despite their great potential, the availability of FAPCs is limited by tedious synthetic schemes including multiple reaction steps. ${ }^{5}$

A similar and relevant comparison of synthetic challenges can be correlated to functional and monodisperse dendrimers. More specifically, the family of aliphatic polyesters is based on

KTH Royal Institute of Technology, Department of Fibre and Polymer Technology, Teknikringen 56-68, SE-100 44 Stockholm, Sweden. E-mail: malkoch@kth.se; Fax: +468790 8283; Tel: +4687908768

$\dagger$ Electronic supplementary information (ESI) available: Detailing the experimental procedures and synthesis. See DOI: 10.1039/c4py00911h the simple $\mathrm{AB}_{2}$ monomer 2,2-bis(methylol) propionic acid (bisMPA). From the first synthetic report of a relevantly simple dendrimer, isolated in $\mathrm{mg}$ scales, to large scale simplified approaches accomplished in one day, ${ }^{6}$ the bis-MPA monomer has passed a cascade of synthetic refinements, including click chemistry concepts. ${ }^{7}$ Today, a myriad of structurally different dendritic architectures based on bis-MPA is accessible and exploited in a broad range of application-driven research. ${ }^{7 a}$ With excellent biocompatible, nontoxic and biodegradable properties, their scaffolding evaluation is heavily focused towards the field of biomedicine, especially as promising drug delivery systems. ${ }^{8}$ In the context of PCs, Fréchet and coworkers described a novel synthetic strategy to end-heterofunctional bis-MPA dendrimers utilizing the covalently attached cyclic carbonate bis-MPA monomer, 5-methyl-5-carboxyl-1,3-dioxane2-one (MCC) at the periphery of the framework. ${ }^{9}$ This was accomplished by successful carbonylation of the pendant 1,3diols groups of the MCC. Subsequent amine-induced ring opening of the carbonate-functional dendrimer resulted in the desired peripheral heterofunctionality. ${ }^{9}$ Notably, functional cyclic carbonates, including MCC, are orthogonal in nature and can undergo selective ring opening polymerization (ROP) resulting in FAPCs. Due to their attractive biomedical properties, cationic amphiphilic nanoparticles based on FAPCs were recently found to exhibit excellent antimicrobial effects on bacteria by microbial wall/membrane disruption mechanisms, thus hindering the growth of bacteria such as methicillin-resistant Staphylococcus aureus (MRSA). ${ }^{10}$ As FAPCs continue to migrate into various research fields, a critical challenge to overcome this is improving available synthetic pathways. In this context, Hedrick and co-workers ${ }^{11}$ reported on the synthesis of bench-stable pentafluorophenyl substituted bis-MPA and 1,1,1-tris(hydroxymethyl)ethane (TME). Their findings utilized a tandem carbonylation/pendant-group activation mechanism, thus avoiding tedious protection group chemistry. This strategy is today recognized as the most versatile approach to fast and efficient generation of a variety of functional carbonates. Unfortunately, the fluorinated reagent is still rather expensive, resulting in functional carbonates 
mostly limited to gram scales. Therefore, further simplification of the synthetic methodology is inevitable to further facilitate the availability of these significant and functional buildingblocks on larger synthetic scales.

We herein present a robust and economical synthetic protocol for the synthesis of functional cyclic carbonates utilizing 1,1'-carbonyldiimidazole (CDI) as the reagent. Incentives for using CDI include its ready availability, low price and safety for handling and storage. Previously, CDI has been widely used as a reagent for formation of urethane, urea, ester and amide-linkages. ${ }^{12}$ It has also been exploited for synthesis of peptides, ${ }^{13,14}$ dendritic polymers, ${ }^{15}$ cellulose surfaces, ${ }^{16}$ and even for 5- and 6-membered cyclic carbonates. ${ }^{17,18}$ The described robust and operationally facile methodology was specifically targeting functional 6-membered cyclic carbonate derivatives based on $\mathrm{A}_{3}$ and $\mathrm{AB}_{2}$ monomers, more specifically trimethylolpropane (TMP) and bis-MPA. An overview of the concept including the polymerization to FAPCs is described in Scheme 1, and capitalizes on the isolation of two critical and reactive imidazole-functional intermediates 1 and 2. Conversion of 1 and 2 by alcohol-mediated imidazole displacement was utilized for the synthesis of a diverse library of functional cyclic carbonates based on TMP and bis-MPA (Fig. 1).

From initial screening experiments, we found that reaction of 1 eq. of TMP together with 2 eq. of CDI in acetone resulted in a spontaneous precipitation of TMP imidazole carbonate (TMPIC) 1. After a simple filtration, TMPIC 1 could be isolated

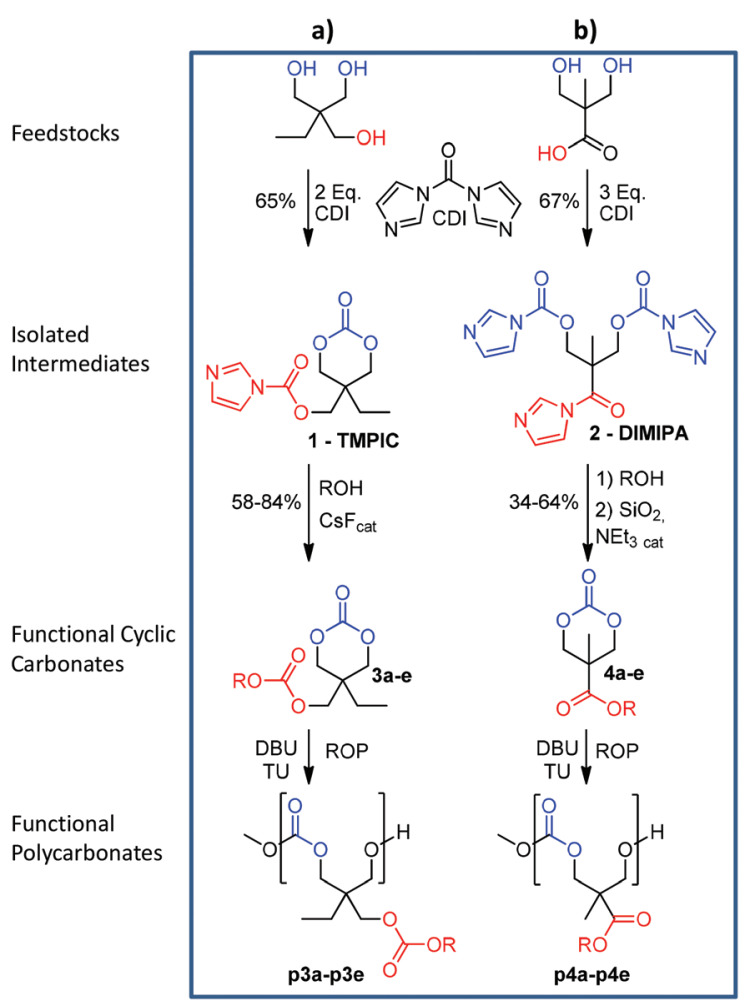

Scheme 1 Synthetic outline for accelerated synthesis of functional carbonate monomers and polymers from readily available TMP (a) and bis-MPA (b) feedstocks.

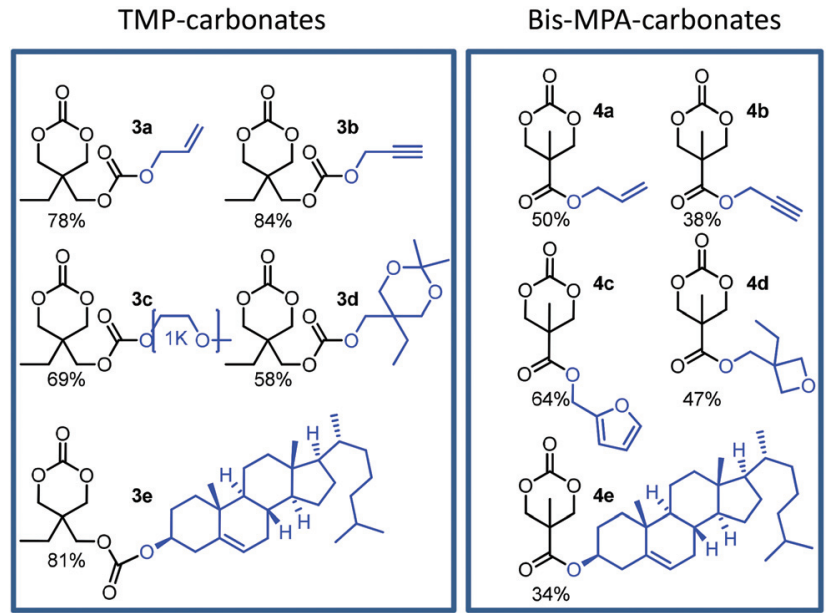

Fig. 1 Synthesized library of functional cyclic carbonates.

as a white solid in $65 \%$ yield on an initial $>60$ gram scale. In contrast, treatment of bis-MPA with 2 eq. of CDI in acetone under similar conditions did not yield the corresponding cyclic carbonate product. Interestingly, increasing the ratio between CDI and bis-MPA to 3:1 in THF resulted in spontaneous formation of a white solid. After a simple filtration procedure, tris-imidazole functional bis-MPA 2 (DIMIPA) was isolated in $67 \%$ yield on a close to 100 gram scale. ${ }^{1} \mathrm{H}-\mathrm{NMR}$ spectra of TMPIC 1 and DIMIPA 2 can be seen in Fig. 2a and $3 \mathrm{a}$, respectively. Both reactive intermediates could be stored on the bench for months without any indication of degradation.

As an evident next step, a robust and selective postfunctionalization strategy was sought out for TMPIC 1 and DIMIPA 2 towards libraries of cyclic carbonates bearing relevant pendant functional groups. After initial screening of reaction conditions, we found that selective and near complete substitution of the imidazole group of TMPIC 1 with various alcohols was accomplished in acetone using $1 \mathrm{~mol} \%$ of cesium fluoride

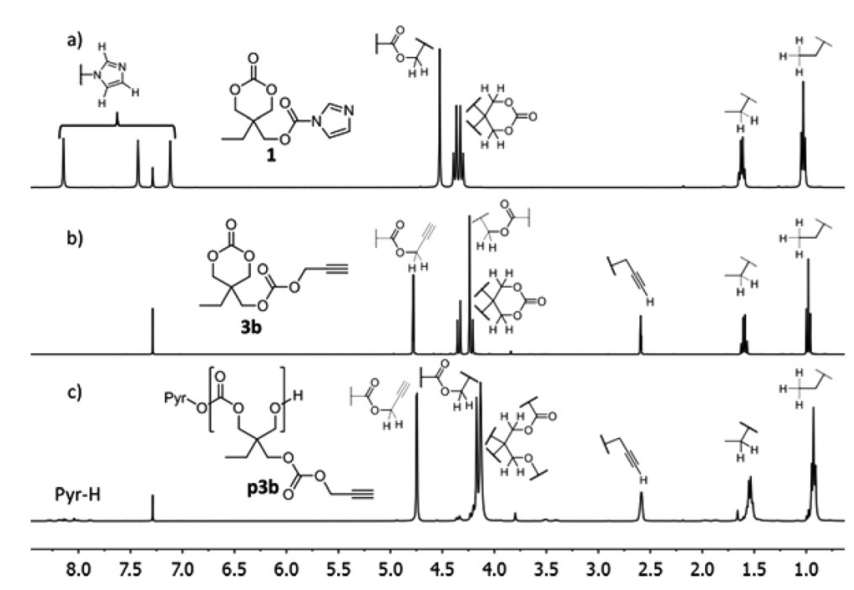

Fig. $2{ }^{1} \mathrm{H}-\mathrm{NMR}\left(\mathrm{CDCl}_{3}\right)$ spectra of (a) imidazole-TMP-carbonate 1, (b) propargyl-TMP-carbonate $3 \mathrm{~b}$, and (c) poly propargyl-TMP-carbonate p3b. 


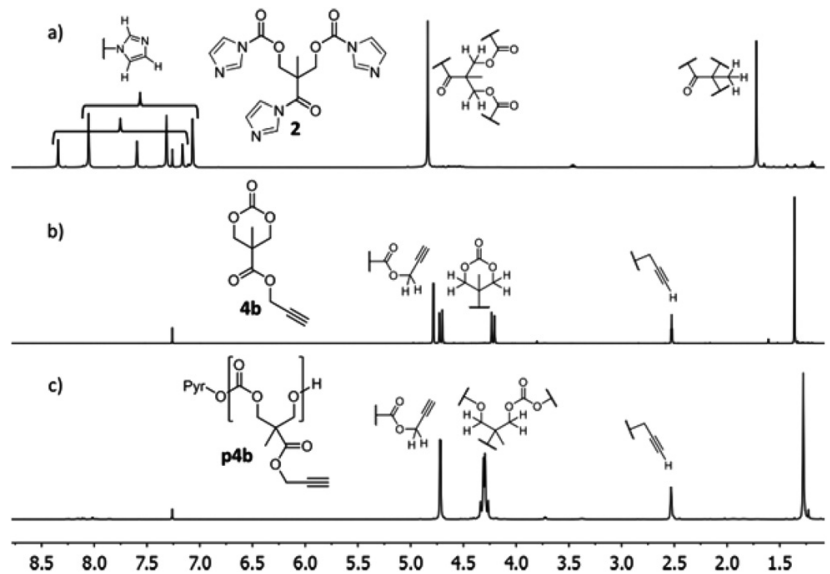

Fig. $3{ }^{1} \mathrm{H}-\mathrm{NMR}\left(\mathrm{CDCl}_{3}\right)$ spectra of (a) triimidazole-bis-MPA-carbonate 2, (b) propargyl-bisMPA-carbonate $\mathbf{4 b}$, and (c) poly propargyl-bis-MPAcarbonate $\mathrm{p} 4 \mathrm{~b}$.

(CsF), resulting in functional TMP-based cyclic carbonates 3a-e (Scheme 1, Fig. 1).

An illustrative example of the ${ }^{1} \mathrm{H}-\mathrm{NMR}$ spectra of TMPIC 1 and products from reaction with propargyl alcohol into propargyl-TMP-carbonate $\mathbf{3} \mathbf{b}$, and final ROP polymerization into $\mathbf{p} 3 \mathbf{b}$ are shown in Fig. 2.

A clear shift movement of the $-\mathrm{CH}_{2}$ protons in the carbonate ring was found, from 4.25 and $4.38 \mathrm{ppm}$ in 1 to 4.20 and $4.31 \mathrm{ppm}$ in $\mathbf{3 b}$. Similar conditions for postfunctionalization by catalytic amounts of CsF when using DIMIPA 2 as the precursor did not produce the corresponding cyclic carbonates in any acceptable yield. Interestingly, conversion of 2 was instead achieved using a one-pot two-step procedure involving selective alcohol-induced displacement of the carboxylic imidazolyl group to produce an ester linkage, followed by a silica $\left(\mathrm{SiO}_{2}\right)$ mediated ring-closing process in the presence of triethylamine (TEA) yielding bis-MPA based cyclic carbonate monomers 4a-e, Scheme 1 b and Fig. 1 . The ${ }^{1} \mathrm{H}-\mathrm{NMR}$ spectrum of 2 clearly shows a singlet corresponding to the $-\mathrm{CH}_{2}$ bis-MPA protons in DIMIPA at $4.84 \mathrm{ppm}$, which upon propargyl alcohol induced substitution and carbonate ring formation to $\mathbf{4 b}$ moves into two doublets at 4.21 and 4.69 ppm, Fig. 3a and b. Subsequent ROP of $\mathbf{4 b}$ into $\mathbf{p} \mathbf{4 b}$ leads to a merging of the doublet signals, Fig. 3c.

Acetone was found to be the best solvent for the transformation of $\mathbf{1}$ and $\mathbf{2}$ to their respective cyclic carbonates, possibly due to a stabilizing effect upon the formed cyclic carbonate monomers. The use of other organic solvents, such as dichloromethane and THF, was accompanied by significant side reactions like alcohol-induced ring-opening or oligomerization processes. Also, workup by silica filtration of the $\mathrm{Et}_{2} \mathrm{O}$-diluted $(1: 1)$ crude reaction mixtures prior to concentration in vacuo was proven essential to avoid oligomerization side-reactions, probably due to removal of the residual CsF catalyst and the imidazole byproduct.

As seen in Fig. 1, the described synthetic protocols enable the construction of aliphatic cyclic monomers with high func- tional group tolerance and selectivity. The displayed carbonates were carefully chosen to cover a range of relevant functionalities being bio- and bulky hydrophilic (MeO-PEG, 3c) or hydrophobic (cholesterol 3e,4e) as well as reactive. For the latter, cyclic carbonate monomers containing allyl- ${ }^{19}$ $(\mathbf{3 a}, \mathbf{4 a})$, propargyl- ${ }^{20}(\mathbf{3 b}, \mathbf{4 b})$, oxetane (4d) and furfuryl-(4c) pendant functionalities are envisioned highly valuable, due to well established post-functionalization protocols by click reactions. ${ }^{5,21}$ Also, the utility of oxetane- ${ }^{22}$ and allyl ${ }^{23}$-functional polymers for efficient crosslinking into polymeric networks have been demonstrated. All reactions were typically conducted on a 10 gram scale and monomers were isolated using standard chromatographic procedures. Without any further optimization, the overall yields of cyclic carbonates based on TMP (3a-e) were between 58 and $84 \%$ and $34-64 \%$ for the bis-MPA based (4a-e). The purity of all cyclic carbonates was corroborated by ${ }^{1} \mathrm{H}-\mathrm{NMR},{ }^{13} \mathrm{C}-\mathrm{NMR}$ and high-resolution mass spectrometry (HRMS), as can be found in the ESI (S1-S24). $\dagger$

Satisfied with the simplicity and scalability of the CDI strategy, the functional cyclic carbonates were subjected to ROPs to deliver a library of FACPs using standard organocatalytic conditions. ${ }^{24}$ The targeted molecular weight for all polymers was set to $10 \mathrm{kDa}$ using pyrenebutanol as an initiator together with $5 \mathrm{~mol} \%$ of the $1: 1$ molar ratio between 1,8-diazabicyclo-5.4.0undec-7-ene (DBU) and 1-(3,5-bis(trifluoromethyl)phenyl3-cyclohexyl-2-thiourea (TU), Scheme 1 and Table 1.

For the TMP-functional cyclic carbonates, the polymerization was found to proceed to their maximum conversion within 16 hours, producing polycarbonates with bulky functionalities, i.e. cholesterol p3e (42\%) and PEG p3c (66\%) to clickable propargyl p3b (89\%). The ${ }^{1} \mathrm{H}-\mathrm{NMR}$ spectrum of the latter can be seen in Fig. 2c. Additionally, while ${ }^{1} \mathrm{H}-\mathrm{NMR}$ analysis detail molecular weights of $\mathbf{p 3 a}-\mathbf{p} 3 \mathbf{e}$ close to the targeted values the results from SEC were notably lower and with dispersities $(\nexists)$ ranging from 1.3 to 2.1 , Fig. S35 (ESI). $\dagger$ This is in line with previous observations by Hedrick and co-workers on

Table 1 ROP of functional cyclic carbonates ${ }^{a}$

\begin{tabular}{llllllll}
\hline & $\begin{array}{l}\text { Time } \\
\text { M }\end{array}$ & M/I & $\begin{array}{l}\operatorname{Conv}^{b} \\
(\%)\end{array}$ & $\begin{array}{l}M_{\mathrm{n}} \mathrm{SEC}^{c} \\
(\mathrm{kDa})\end{array}$ & $\begin{array}{l}M_{\mathrm{n}} \mathrm{NMR}^{d} \\
(\mathrm{kDa})\end{array}$ & $\begin{array}{l}M_{\mathrm{n}} \text { theo }^{e} \\
(\mathrm{kDa})\end{array}$ & $\Xi^{c}$ \\
\hline 3a & 6 & 50 & 82 & 3.4 & 10.8 & 10.3 & 1.7 \\
3b & 6 & 50 & 89 & 3.7 & 11.2 & 10.9 & 1.9 \\
3c & 16 & 10 & 63 & 6.6 & 9.9 & 7.8 & 2.1 \\
3d & 16 & 50 & 64 & 4.4 & 13.0 & 11.8 & 1.3 \\
3e & 16 & 20 & 42 & n.d. ${ }^{f}$ & 4.8 & 11.7 & n.d. \\
4a & 2 & 50 & 89 & 5.8 & 11.2 & 9.0 & 1.3 \\
4b & 2 & 50 & 99 & 10.5 & 10.8 & 10.0 & 1.4 \\
4c & 2 & 50 & 93 & 4.5 & 11.8 & 11.3 & 1.4 \\
4d & 2 & 50 & 99 & 8.2 & 8.3 & 13.1 & 1.3
\end{tabular}

${ }^{a}$ All reactions were conducted in sealed vials, under an $\mathrm{N}_{2}$-atm. For all polymerization reactions, a $5 \mathrm{~mol} \%$ catalytic loading of (DBU-TU) 1:1 and pyrenebutanol (PyrOH) initiator was used in dichloromethane solution, with a monomer concentration of $1 \mathrm{M}$ at room temperature. ${ }^{b}$ Crude ${ }^{1} \mathrm{H}-\mathrm{NMR}$ analysis. ${ }^{c} \mathrm{SEC}$ (DMF) with PMMA calibration. ${ }^{d 1} \mathrm{H}$-NMR end-group integration. ${ }^{e}$ Based on measured monomer conversion. ${ }^{f}$ Not detected due to formation of assembled aggregates. 
ROP using similar monomers. ${ }^{11}$ Interestingly, FAPC with the pendant cholesterol group p3e was found to form strong intermolecular agglomerations and thus unsuccessful detection by SEC. No further trials were attempted to increase the conversions of 3a-e.

In contrast, ROPs of bis-MPA carbonates $\mathbf{4 a - d}$, under similar conditions to the TMP-based counterparts proceeded to high conversions (89-99\%) after 2 hours at room temperature. The ${ }^{1} \mathrm{H}-\mathrm{NMR}$ spectrum of the purified propargyl functional bis-MPA polycarbonate $\mathbf{p} \mathbf{4 b}$ can be found in Fig. $3 \mathrm{c}$ with distinct shift of the $-\mathrm{CH}_{2}$ upon ring-opening. Also, Table 1 and Fig. S36 (ESI) $\uparrow$ display the obtained molecular weights by SEC which were in closer agreement to the theoretical ones and with lower observed $D$ of 1.3-1.4. The thermal behavior of a number of both carbonate families bearing allyl- and propargyl-pendant groups was investigated by differential scanning calorimetry (DSC) (see ESI $\dagger$ for details). The polymers were found to display similar glass transition temperatures, independently of the TMP or bis-MPA core carbonate structure. For both bisMPA- and TMP-functional PCs (p3a and p4a), the $T_{\mathrm{g}}$ 's were noted to be $-12^{\circ} \mathrm{C}$, whereas the $T_{\mathrm{g}}$ of the corresponding propargyl functional PCs were $+7{ }^{\circ} \mathrm{C}(\mathbf{p} 3 \mathbf{b})$ and $+9{ }^{\circ} \mathrm{C}(\mathbf{p} 4 \mathbf{b})$, respectively, collected on the second heating scan at $10{ }^{\circ} \mathrm{C}$ $\min ^{-1}$ heating and cooling rate.

With respect to the envisioned potential biomedical applications of these functional polycarbonates, the cytotoxicity of the key reactive imidazole intermediates $\mathbf{1}$ and 2 , the subsequent monomers and their corresponding polymers p3a-e and $\mathbf{p 4 a - e}$ were tested according to ISO10993-5 procedures. $^{25}$ Human dermal fibroblasts (hDFs), being the most abundant cells in human dermis were chosen for the initial cytotoxicity. This as the interaction between hDFs and biomaterials, such as wound dressings, is highly relevant in skin regeneration applications. Fig. 4 details the cell viability of human dermal

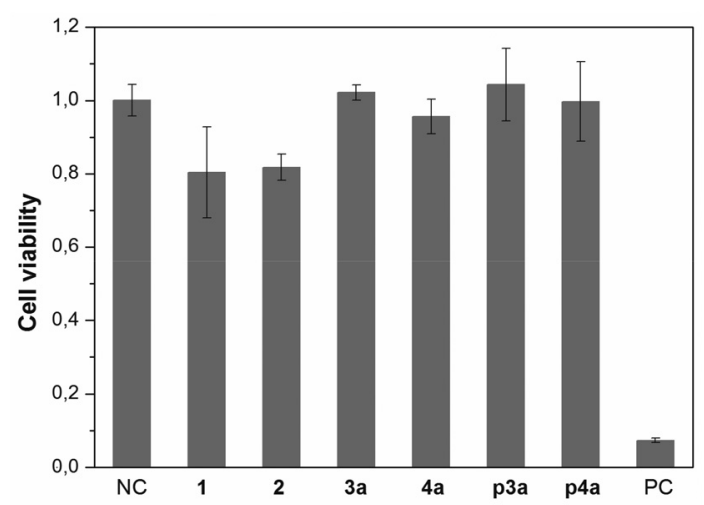

Fig. 4 Cell viability of hDF after culturing in extracts of cell culture coverslips coated with $0.1 \mathrm{mg}$ of the intermediates 1 and 2, the monomers $3 a$ and $4 a$ and the polymers $\mathrm{p} 3 \mathrm{a}$ and $\mathrm{p} 4 \mathrm{a}$. Extract of uncoated coverslips was employed as negative control (NC) to define cell viability of 1. Cell culture with addition of $0.1 \%$ Triton $X-100$ served as the positive control (PC). Cell viability was evaluated after culturing in an incubator at $37{ }^{\circ} \mathrm{C}$ and $5 \% \mathrm{CO}_{2}$ in a humidified atmosphere for $24 \mathrm{~h}$. The data represent the mean \pm standard deviation of three replicates. fibroblasts (hDF) cultured in complete growth medium (CGM) extract of materials resulting in allyl functional PCs.

Both carbonate monomers $\mathbf{3 a}$ and $\mathbf{4 a}$ and polymers (p3a and p4a) showed no toxicity, i.e. no significant difference observed in the metabolic activity of cells compared to that of negative control. The CGM extracts of intermediates ( 1 and 2 ) caused slight reactivity of cells indicated by the decrease of metabolic activity by about $20 \%$. Complete evaluations of all the polycarbonates synthesized in this work (p3a-e and p4a-d) are included in the ESI Fig. S38-S41. $\dagger$ All polycarbonates display low toxicity in elution tests or in the direct contact tests, in which the hDF cells were cultured directly on the surface of polycarbonate coated coverslips (see $\mathrm{ESI} \dagger$ for details).

In conclusion, a simplified, robust and cost-efficient synthetic strategy has been identified which unlocks the availability of an array of functional cyclic carbonate monomers at large scale. The methodology capitalised on the facile use of CDI as a reagent and which together with simple $A_{2}$ and $A_{3}$ monomers resulted in the isolation of stable and reactive imidazole intermediates on a 100 gram scale. In contrast to previously published procedures, the current synthetic approach delivers functional aliphatic cyclic carbonate monomers and their corresponding FAPCs on large scales which open new opportunities in the design of functional carbonate materials with tailored applications. The relevance of these functional polycarbonate materials for biomedical applications was highlighted by preliminary cytotoxicity studies using human dermal fibroblasts where no indications of adverse behavior were observed.

We acknowledge Knut och Alice Wallenberg Foundation (2012-0196), Marcus Amalia Wallenberg Foundation and the Swedish Research Council VR (2011-5358 and 2010-453) for their financial support.

\section{Notes and references}

1 (a) C. B. Cooley, B. M. Trantow, F. Nederberg, M. K. Kiesewetter, J. L. Hedrick, R. M. Waymouth and P. A. Wender, J. Am. Chem. Soc., 2009, 131, 16401; (b) E. I. Geihe, C. B. Cooley, J. R. Simon, M. K. Kiesewetter, J. A. Edward, R. P. Hickerson, R. L. Kaspar, J. L. Hedrick, R. M. Waymouth and P. A. Wender, Proc. Natl. Acad. Sci. U. S. A., 2012, 109, 13171-13176; (c) J. M. Pelet and D. Putnam, Pharm. Res., 2013, 30, 2166-2166; (d) P. N. Zawaneh, S. P. Singh, R. F. Padera, P. W. Henderson, J. A. Spector and D. Putnam, Proc. Natl. Acad. Sci. U. S. A., 2010, 107, 11014-11019.

2 A. C. Albertsson and M. Eklund, J. Appl. Polym. Sci., 1995, 57, 87-103.

3 K. Fu, D. W. Pack, A. M. Klibanov and R. Langer, Pharm. Res., 2000, 17, 100-106.

4 J. Feng, R. X. Zhuo and X. Z. Zhang, Prog. Polym. Sci., 2012, 37, 211-236. 
5 S. Tempelaar, L. Mespouille, O. Coulembier, P. Dubois and A. P. Dove, Chem. Soc. Rev., 2013, 42, 1312-1336.

6 H. Ihre, A. Hult and E. Soderlind, J. Am. Chem. Soc., 1996, 118, 6388-6395.

7 (a) A. Carlmark, E. Malmström and M. Malkoch, Chem. Soc. Rev., 2013, 42, 5858-5879; (b) M. V. Walter and M. Malkoch, Chem. Soc. Rev., 2012, 41, 4593-4609; (c) P. Antoni, Y. Hed, A. Nordberg, D. Nyström, H. von Holst, A. Hult and M. Malkoch, Angew. Chem., Int. Ed., 2009, 48, 2126-2130.

8 (a) C. C. Lee, J. A. MacKay, J. M. J. Fréchet and F. C. Szoka, Nat. Biotechnol., 2005, 23, 1517-1526; (b) C. C. Lee, E. R. Gillies, M. E. Fox, S. J. Guillaudeu, J. M. J. Frechet, E. E. Dy and F. C. Szoka, Proc. Natl. Acad. Sci. U. S. A., 2006, 103, 16649-16654.

9 A. P. Goodwin, S. S. Lam and J. M. J. Frechet, J. Am. Chem. Soc., 2007, 129, 6994.

10 F. Nederberg, Y. Zhang, J. P. K. Tan, K. J. Xu, H. Y. Wang, C. Yang, S. J. Gao, X. D. Guo, K. Fukushima, L. J. Li, J. L. Hedrick and Y. Y. Yang, Nat. Chem., 2011, 3, 409-414.

11 (a) D. P. Sanders, K. Fukushima, D. J. Coady, A. Nelson, M. Fujiwara, M. Yasumoto and J. L. Hedrick, J. Am. Chem. Soc., 2010, 132, 14724-14726; (b) D. P. Sanders, D. J. Coady, M. Yasumoto, M. Fujiwara, H. Sardon and J. L. Hedrick, Polym. Chem., 2014, 5, 327-329; (c) A. C. Engler, J. M. W. Chan, D. J. Coady, J. M. O'Brien, H. Sardon, A. Nelson, D. P. Sanders, Y. Y. Yang and J. L. Hedrick, Macromolecules, 2013, 46, 1283-1290.

12 R. K. Sharma, Synlett, 2007, 3073-3074.

13 K. W. Ehler and L. E. Orgel, Biochim. Biophys. Acta, 1976, 434, 233-243.

14 X. W. Zhang, J. Rodrigues, L. Evans, B. Hinkle, L. Ballantyne and M. Pena, J. Org. Chem., 1997, 62, 6420-6423.
15 (a) S. P. Rannard and N. J. Davis, J. Am. Chem. Soc., 2000, 122, 11729-11730; (b) S. P. Rannard, N. J. Davis and I. Herbert, Macromolecules, 2004, 37(25), 9418-9430.

16 S. Alila, A. M. Ferraria, A. M. B. do Rego and S. Boufi, Carbohydr. Polym., 2009, 77, 553-562.

17 S. P. Rannard and N. J. Davis, Org. Lett., 1999, 1, 933-936.

18 J. Mindemark and T. Bowden, Polymer, 2011, 52, 5716-5722.

19 S. Tempelaar, L. Mespouille, P. Dubois and A. P. Dove, Macromolecules, 2011, 44, 2084-2091.

20 S. Tempelaar, I. A. Barker, V. X. Truong, D. J. Hall, L. Mespouille, P. Dubois and A. P. Dove, Polym. Chem., 2013, 4, 174-183.

21 (a) W. H. Binder and R. Sachsenhofer, Macromol. Rapid Commun., 2007, 28, 15-54; (b) P. S. Omurtag, U. S. Gunay, A. Dag, H. Durmaz, G. Hizal and U. Tunca, J. Polym. Sci., Part A: Pol. Chem., 2013, 51, 2252-2259; (c) M. Malkoch, R. J. Thibault, E. Drockenmuller, M. Messerschmidt, B. Voit, T. P. Russell and C. J. Hawker, J. Am. Chem. Soc., 2005, 127, 14942-14949.

22 C. D. Muller, A. Falcou, N. Reckefuss, M. Rojahn, V. Wiederhirn, P. Rudati, H. Frohne, O. Nuyken, H. Becker and K. Meerholz, Nature, 2003, 421, 829-833.

23 S. M. Trey, C. Nilsson, E. Malmstrom and M. Johansson, Prog. Org. Coat., 2010, 67, 348-355.

24 (a) N. E. Kamber, W. Jeong, R. M. Waymouth, R. C. Pratt, B. G. Lohmeijer and J. L. Hedrick, Chem. Rev., 2007, 107, 5813-5840; (b) A. P. Dove, R. C. Pratt, B. G. G. Lohmeijer, R. M. Waymouth and J. L. Hedrick, J. Am. Chem. Soc., 2005, 127, 13798-13799.

25 (a) X. L. Wang, R. X. Zhuo, S. W. Huang, L. J. Liu and F. He, Macromol. Chem. Phys., 2002, 203, 985-990; (b) R. Zange, Y. X. Li and T. Kissel, J. Controlled Release, 1998, 56, 249258; (c) ISO 10993-5: Biological evaluation of medical devices - Part 5: Tests for in vitro cytotoxicity, 2009. 